\title{
Application of the finite volume method for the supersonic flow around the axisymmetric cone body placed in a free stream
}

\author{
R. Haoui \\ Department of Mechanical Engineering, \\ University of Science and Technology, Bab Ezzouar, Algeria
}

\begin{abstract}
The aim of this study is to determine the supersonic flow parameters around the axisymmetric cone body by finite volume methods. A code is written to capture the oblique shock wave behind a cone placed in supersonic free stream. The numerical method uses the Flux Vector Splitting method of Van Leer (Flux Vector Splitting for the Euler Equations, Lecture Notes in Physics, 170, pp. 507$512,1982)$. Time stepping is used as a parameter to ensure the convergence of the solution. The CFL coefficient and the mesh size are the other two parameters used to steady the convergence (Haoui et al. Condition de convergence appliquée à un écoulement réactif axisymétrique, 16ème CFM, n 738, Nice, France, 2003). The shock wave is detached when the point angle is large or the Mach number is weak. The Mach-contours show the evolution of the flow well from the infinite one to after the body. The precision of calculations is an order $10^{-8}$. For the same infinite Mach number, when the point angle increases, the detached shock back away. The computer code also collects also the waves of relaxation on the convex part of the body.
\end{abstract}

Keywords: axisymmetric, supersonic flow, cone body, finite volume, oblique shock wave.

\section{Introduction}

A numerical technique is proposed to predict a supersonic flow around an axisymmetric cone body. An explicit formulation with the finite volume method 
is used for this purpose. The numerical method uses the split flux schemes. This procedure is sufficient because it gives excellent results with the experimental values and the convergence is assured with a high precision. The oblique shock is attached to the cone where a sudden increase of the pressure takes place. The aim is to determine the pressure distribution around the cone body model and the variation for the angle of the shock in function of the Mach number and the inclination of cone point angle. Fig. 1 shows the setup of the cone and the computational domain.

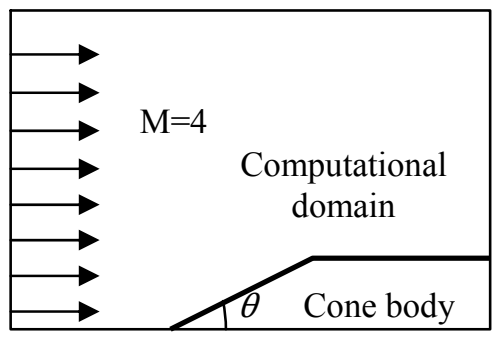

Figure 1: Configuration of the cone body $\theta=20^{\circ}$.

\section{Equations}

The basic equations are those of Euler written in a 3D vector form as follow:

$$
\frac{\partial W}{\partial t}+\operatorname{div}(f)=\Omega
$$

where $\vec{f}=F \cdot \vec{\imath}+G \cdot \vec{\jmath}+H \cdot \vec{k}, W$ and $\Omega$ are:

$$
\begin{array}{ccc}
W=\left(\begin{array}{c}
\rho \\
\rho u \\
\rho v \\
\rho w \\
\rho e
\end{array}\right) \quad F=\left(\begin{array}{c}
\rho u \\
\rho u^{2}+p \\
\rho u v \\
\rho u w \\
(\rho e+p) u
\end{array}\right) \quad G=\left(\begin{array}{c}
\rho v \\
\rho u v \\
\rho v^{2}+p \\
\rho v w \\
(\rho e+p) v
\end{array}\right) \\
H=\left(\begin{array}{c}
\rho w \\
\rho u w \\
\rho v w \\
\rho w^{2}+p \\
(\rho e+p) w
\end{array}\right) & \Omega=\left(\begin{array}{l}
0 \\
0 \\
0 \\
0 \\
0
\end{array}\right)
\end{array}
$$

This system of equations is closed by the equation of state, $p=\rho r T$ and the non-linear equation $e=f(T)$. Where the specific total energy is:

$$
e=c_{v} T+\frac{1}{2}\left(u^{2}+v^{2}\right)
$$


The first term corresponds to the translation-rotation internal energy and the second term to the kinetic energy.

\section{Method of resolution}

The unsteady non-linear PDE's are solved using an explicit form. The time is considered as an iterative parameter. The steady state solution is obtained when the residue of relative variation of density is about $10^{-8}$. The two-dimensional axisymmetric form is obtained by using the perturbation method to the threedimensional flow problem, (Haoui et al. [2]). Fig. 2 shows the mesh form used in the computational domain.

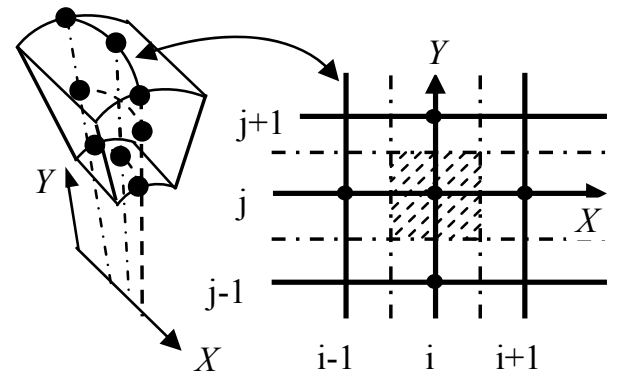

Figure 2: Mesh form.

We obtain the system:

$$
\begin{gathered}
\operatorname{mes}\left(C_{i, j}\right) \frac{\partial W_{i, j}}{\partial t}+\sum_{a \in\left\{x, x^{\prime}, y, y^{\prime}\right\}}\left(F_{i, j} \vec{\imath}+G_{i, j} \vec{\jmath}\right) \overrightarrow{\eta_{a}}- \\
2 p\left(\begin{array}{l}
0 \\
0 \\
1 \\
0
\end{array}\right) . \operatorname{aire}\left(C_{i, j}\right)=\operatorname{mes}\left(C_{i, j}\right) \cdot \Omega_{\mathrm{i}, \mathrm{j}}
\end{gathered}
$$

or

$$
\begin{gathered}
W_{i, j}^{n+1}=W_{i, j}^{n}-\frac{\Delta t_{i, j}}{\operatorname{mes}\left(C_{i, j}\right)} \sum_{a \in\left\{x, x^{\prime}, y, y^{\prime}\right\}}\left(F_{i, j} \vec{\imath}+G_{i, j} \vec{\jmath}\right) \overrightarrow{\eta_{a}}+ \\
\Delta t_{i, j} \frac{\operatorname{aire}\left(C_{i, j}\right)}{\operatorname{mes}\left(C_{i, j}\right)} H_{i, j}+\Delta t_{i, j} \Omega_{\mathrm{i}, j}
\end{gathered}
$$

where

$$
\Delta t_{i, j}=\min \frac{\Delta x . C F L}{\left\|V_{i, j}\right\|+a_{i, j}}, \quad\left\|V_{i, j}\right\|=\sqrt{u^{2}+v^{2}}
$$

$\Delta x$ is the minimum mesh size. For mes $(i, j)$ and aire $(i, j)$ see [2]. 
In this study, we chose to use a breakdown of flows into two parts $f_{V L}^{-}$et $f_{V L}^{+}$. This decomposition should apply to our problem which is two-dimensional, where we must calculate the flow at each interface between two cells. However, through this interface, the direction that is most important is the normal $\vec{\eta}$. Therefore, we change the reference for placing in the benchmark of the interface as the normal reference through a rotation vector $R$ (fig. 3). The flux vector $W_{E}$ can be written $W_{E}^{R}$ in the new benchmark:

$$
W_{E}^{R}=\left(\begin{array}{c}
\rho \\
\rho \overrightarrow{V_{n}} \\
\rho e
\end{array}\right)
$$

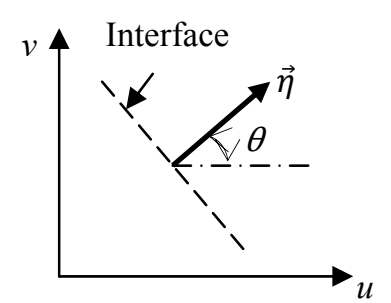

Figure 3 : Interface with the normale.

where $\overrightarrow{V_{n}}$ is obtained from $\vec{V}$, through the rotation $R$, as follows:

where :

$$
\vec{V}=\left(\begin{array}{l}
u \\
v
\end{array}\right) \rightarrow \overrightarrow{V_{n}}=\left(\begin{array}{l}
u_{n} \\
v_{n}
\end{array}\right)=\left(\begin{array}{cc}
\cos \theta & \sin \theta \\
-\sin \theta & \cos \theta
\end{array}\right)\left(\begin{array}{l}
u \\
v
\end{array}\right)
$$

$$
\cos \theta=\frac{\eta_{x}}{\|\vec{\eta}\|}, \quad \sin \theta=\frac{\eta_{y}}{\|\vec{\eta}\|}, \quad\|\vec{\eta}\|=\sqrt{\eta_{x}^{2}+\eta_{y}^{2}}
$$

The transformation $R$ is written broadly as

$$
R=\left(\begin{array}{cc}
\cos \theta & \sin \theta \\
-\sin \theta & \cos \theta
\end{array}\right) \quad \text { et } \quad R^{-1}=\left(\begin{array}{cc}
\cos \theta & -\sin \theta \\
\sin \theta & \cos \theta
\end{array}\right)
$$

In addition, each interface $i+1 / 2$, we know the two neighboring states $i$ and $i+1$. We can thus calculate the flow $\mathrm{F}$ through the interface, the overall flow $f(W, \vec{\eta})$ being deducted from $F$ by applying the inverse of the rotation:

$$
f(W, \vec{\eta})=\|\vec{\eta}\| \cdot R^{-1}\left(F\left(W^{R}\right)\right)
$$

This property makes it possible to use only one component of flux $f$ ( $F$, for example) to determine the breakdown of two-dimensional flows. In addition, this method is easier to implement and less expensive than the breakdown of twodimensional flux $f=F \eta_{x}+G \eta_{y}$. The expressions of $F_{V L}^{+}$and $F_{V L}^{-}$in 1-D 
which are those of $F_{V L}^{+}\left(W^{R}\right)$ and $F_{V L}^{-}\left(W^{R}\right)$, where $W^{R}$ is defined as the $W$ transformed by the rotation $R$, can be written in the following manner:

$$
F_{V L}^{+}\left(W^{R}\right)=\left\{\begin{array}{cc}
F\left(W^{R}\right) & M_{n} \geq 1 \\
\left(\begin{array}{c}
\frac{\rho c}{4}\left(\frac{u}{c}+1\right)^{2}=f_{1}^{+} \\
\frac{f_{1}^{+}}{\gamma}[(\gamma-1) u+2 c] \\
\frac{f_{1}^{+}}{2} \frac{[(\gamma-1) u+2 c]^{2}}{\gamma^{2}-1}+v^{2}
\end{array}\right) & \left|M_{n}\right|<1 \\
0 & F_{V L}^{-}\left(W^{R}\right)=\left\{\begin{array}{cc}
0 \\
\left(\begin{array}{c}
\frac{\rho c}{4}\left(\frac{u}{c}-1\right)^{2}=f_{1}^{-} \\
\frac{f_{1}^{-}}{\gamma}[(\gamma-1) u-2 c] \\
\frac{f_{1}^{-}}{2} \frac{[(\gamma-1) u-2 c]^{2}}{\gamma^{2}-1}+v^{2}
\end{array}\right) \\
F\left(W^{R}\right) & M_{n} \geq 1
\end{array}\right. \\
\end{array}\right.
$$

where $M_{n}=\frac{u}{c}$

\section{Results and discussion}

Fig. 4 shows a configuration of $(59 \times 51)$ nodes. In our calculation, a refined mesh of $(232 \times 201)$ is used. The CPU time per iteration per mesh point of the perfect gas is found to be $25 \mu \mathrm{s} / \mathrm{iter} / \mathrm{mp}$. The convergence is assured when the residue value equals $10^{-6}$ (fig. 5). In fig. 6 and 7 we show the variation of the Mach number and the pressure distribution along the cone surface. The sudden variation of all parameters confirms the precision of the solution. The number of iterations is found to be around 3000. Figs. 8 and 9 show the Mach-contours around the cone body, for fig. 8 the oblique shocks can be observed. In fig. 9 the cone body is placed in tube shock where we can observe the reflected shock on the wall of shock tube and on the surface cone body. In the case where the semiangle of cone equals $20^{\circ}$, the incident shock angle decreases when the Mach number of the free stream increases. The test values exist in literature and give the shock angle in the function of Mach number of free stream [4]. The shock angle is observed with shadowgraph methods. In table 1, we observe the small difference between the experimental (Shapiro [4]) and computational values (also see fig. 10).

In the case when a semi-angle of cone is chosen to equal $60^{\circ}$, the shock is detached at $\mathrm{M}=2$ (fig. 11). If the Mach number of a free stream increases, the shock is nearer to the cone body. In table 2, we have some examples, also see fig. 12. The mesh grid of $(174 \times 268)$ points and $\mathrm{CFL}=0.4$ are recommended. The number of iterations is about 10000 . 
384 Computational Methods and Experimental Measurements XIV

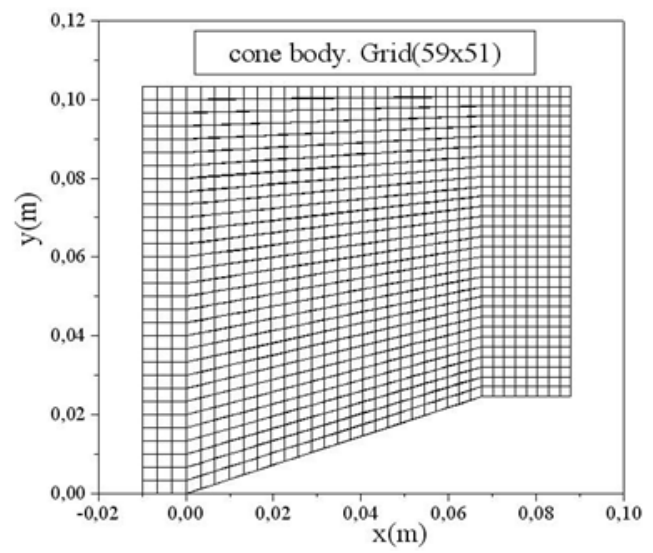

Figure 4: Computational domain.

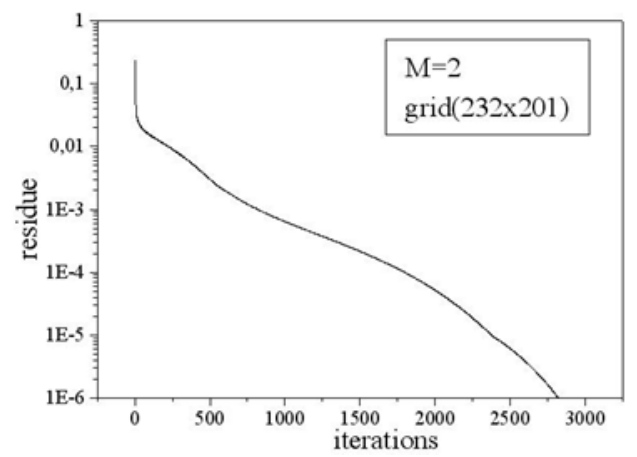

Figure 5: Variation of the residue.

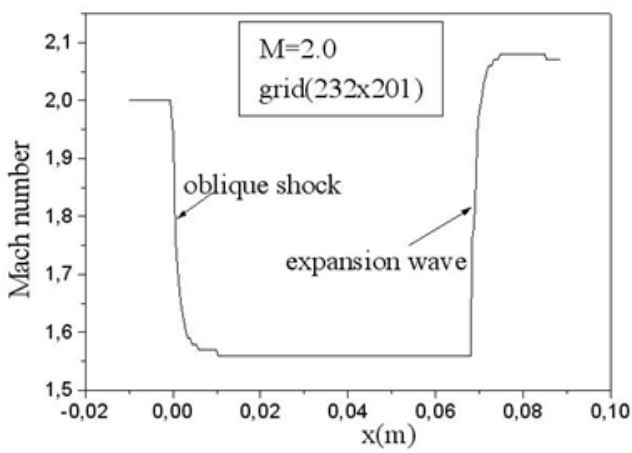

Figure 6: Mach number. 
Computational Methods and Experimental Measurements XIV 385

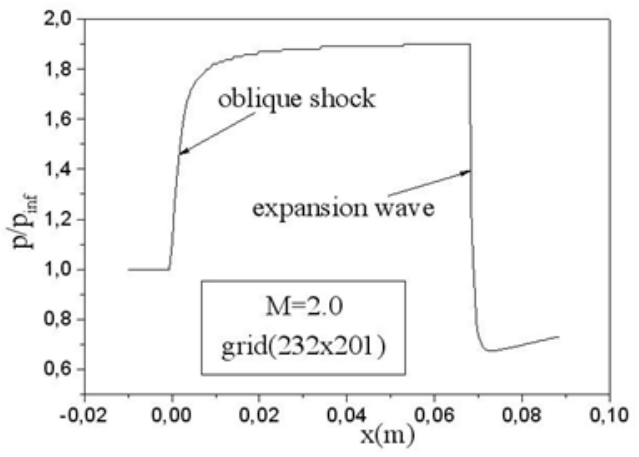

Figure 7: Ratio of pressure distribution.

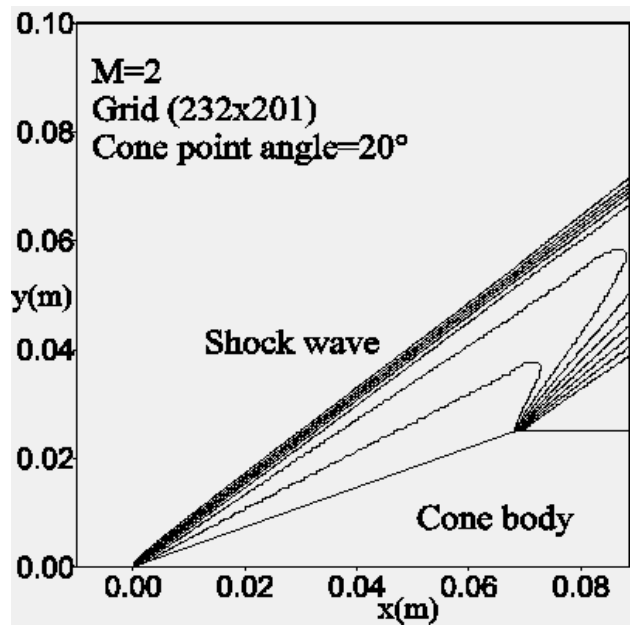

Figure 8: Mach contours.

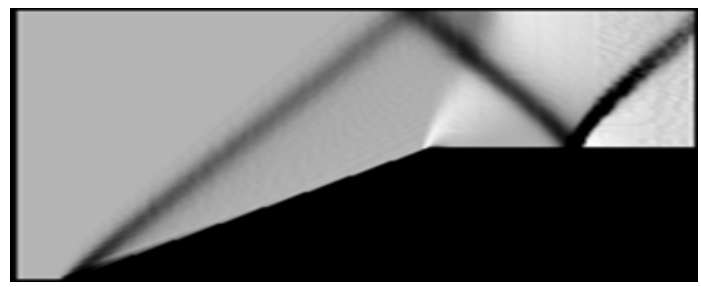

Figure 9: Axisymmetric cone body placed in tunnel. $\mathrm{Mach}=2.0$, angle $=20^{\circ}$. 
Table 1: Attached shock angle for $\theta=20^{\circ}$.

\begin{tabular}{|c|c|c|}
\hline Mach number & computational & experimental \\
\hline 1.4 & $\mathbf{5 3 . 1 3}^{\circ}$ & $53^{\circ}$ \\
\hline 1.6 & $\mathbf{4 6 . 4 0}^{\circ}$ & $46^{\circ}$ \\
\hline 1.8 & $\mathbf{4 2 . 2 7}^{\circ}$ & $42^{\circ}$ \\
\hline 2.0 & $\mathbf{3 8 . 8 8}^{\circ}$ & $39^{\circ}$ \\
\hline
\end{tabular}

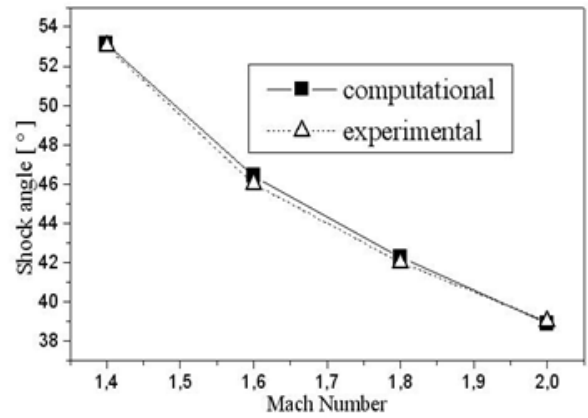

Figure 10: Shock angle.

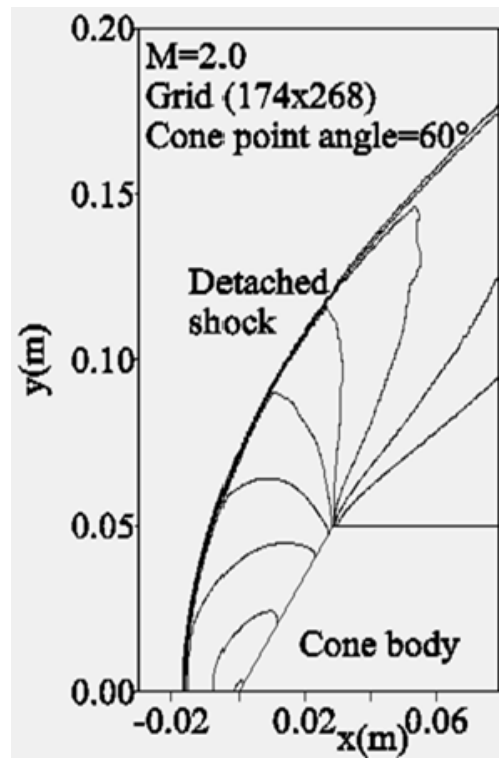

Figure 11: Mach contours. 
Table 2: Detached shock position.

\begin{tabular}{|c|c|c|}
\hline Mach number & Angle $\theta$ & Shock position \\
\hline 2.5 & $45^{\circ}$ & $1 \mathrm{~mm}$ \\
\hline 2.5 & $60^{\circ}$ & $2.7 \mathrm{~mm}$ \\
\hline 2.0 & $45^{\circ}$ & $3 \mathrm{~mm}$ \\
\hline 2.0 & $60^{\circ}$ & $20 \mathrm{~mm}$ \\
\hline 1.5 & $45^{\circ}$ & $4 \mathrm{~mm}$ \\
\hline 1.5 & $60^{\circ}$ & $42 \mathrm{~mm}$ \\
\hline
\end{tabular}

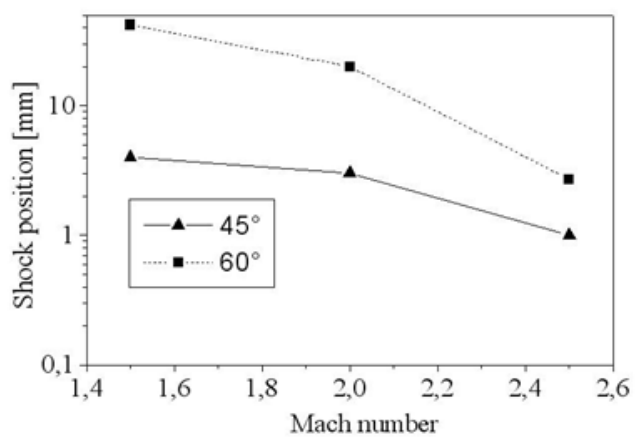

Figure 12: Position of detached shock.

\section{Conclusion}

The finite volume method applied to a supersonic flow problem gives good results and shows the capture of the oblique shocks which are physically true.

The angle of the oblique shock wave depends on the cone point-angle and Mach number of the free-flowing waves. The computational results with the chosen method are in good agreement with experimental observations. Our calculation code was also tested in a case where the cone is placed in the shock tube. The reflected shock on the wall of the tube is clearly visible. When the cone point-angle exceeds a maximum value, the shock wave is detached from the cone body, thus confirming the consistency of the code and the precision of the calculations.

When the angle of the cone point increases, the shock runs away from the blunt body. The respect of boundary conditions especially near the wall plays a decisive role in the accuracy of calculations, especially in the case of the reactive flow around a blunt body. 
388 Computational Methods and Experimental Measurements XIV

\section{References}

[1] Van Leer, B, Flux Vector Splitting for the Euler Equations, Lecture Notes in Physics, 170, pp. 507-512, 1982.

[2] Haoui, R. and al, Condition de convergence appliquée à un écoulement réactif axisymétrique, 16 ème $\mathrm{CFM}, \mathrm{n}^{\circ} 738$, Nice, France, (2003).

[3] Haoui, R., Zeitoun, D. \& Gahmousse, A., Chemical and vibrational nonequilibrium flow in a hypersonic axisymmetric nozzle, IJTS, article 8 , volume 40, pp.787-795, 2001.

[4] Shapiro, A.H., The Dynamics and Thermodynamics of Compressible fluid flow, The Ronald Press Company, New York. Volume II. 664. 1954. 